\title{
Preface
}

\section{Uses and Opportunities for Molecular Imaging in Patients with Breast Cancer}

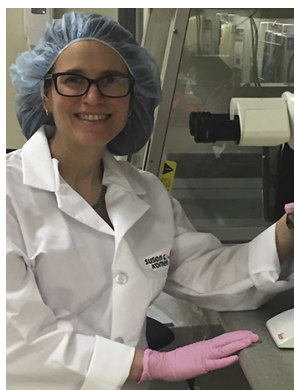

Elizabeth S. McDonald, MD, PhD, FSBI

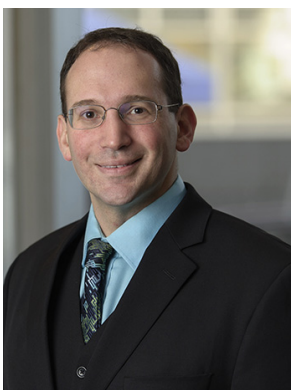

Gary A. Ulaner, MD, PhD, FACNM

Editors

PET has established itself as a clinically valuable imaging method for patients with breast cancer, particularly as hybrid PET/computed tomography (CT) and now as PET/MR. Given the substantial impact of FDG PET and the growing numbers of novel $P E T$ radiotracers in development for patients with breast cancer, it is timely for PET Clinics to review the status of current and future PET imaging modalities for breast malignancy.

This issue of PET Clinics is divided into three sections. The first section covers the principles of breast cancer detection and characterization, which are important for interpretation of PET and other imaging studies. Lebron-Zapata and Jochelson begin the issue with an overview of current breast cancer screening and diagnosis, describing the anatomic and molecular techniques currently in use and the opportunities for molecular and PET techniques to aid in this important aspect of patient care. Next, Provenzano and colleagues summarize the important molecular and histologic subtypes of breast cancer and illustrate how these differences impact the interpretation of PET examinations. Moo and colleagues then summarize the principles of breast cancer therapy, highlighting that while MR has been shown to be effective for surveillance within the breast, other imaging modalities have not demonstrated effective surveillance for recurrence of metastatic disease in asymptomatic patients. This represents an area of tremendous opportunity for PET and other molecular techniques to develop and provide clinical value.

The second section of this issue emphasizes current concepts and successful clinical applications of PET and molecular imaging in patients with breast cancer. FDG is the PET radiotracer that has by far played the most valuable role in PET imaging of breast cancer, including important contributions for the initial systemic staging of patients with locally advanced breast cancer and monitoring of treatment response. These clinical applications of FDG PET are reviewed by Chakraborty and colleagues and Groheux. Dedicated imaging of the breasts by gamma and PET techniques has led to improved molecular imaging of primary breast malignancies. These techniques, their potential clinical applications, and the barriers to widespread adoption of these technologies are summarized by Narayanan and Berg. Bone is the most common site of metastatic disease from breast cancer. Minamimoto and lagaru appraise the roles of molecular and PET imaging for the detection and monitoring of osseous metastases. Sentinel lymph node mapping is an important molecular imaging technique, but as PET imaging does not yet contribute to sentinel node imaging, it was not included as an article in this issue. For a discussion of sentinel node imaging for patients with breast cancer, we suggest a separate excellent recent review by Kim and Zukotynski. ${ }^{1}$ 
The third and final section of this issue addresses the tremendous recent advances in the development of novel PET radiotracers for patients with breast cancer. Hormone receptortargeted PET has a growing range of applications in breast cancer, including assisting early-phase clinical trials of new estrogen receptor (ER)-targeted drug therapies and predicting clinical benefit from these targeted therapies. The developing applications of hormone receptor-targeted PET imaging are discussed by Linden and colleagues. Human epidermal growth factor receptor (HER) family members are the targets of some of the most successful systemic therapies for patients with breast cancer. This makes the potential of HER2- and HER3-targeted imaging an area of considerable interest. These developing applications, including evaluation of heterogeneity of HER2 expression within an individual patient, are presented by Henry and colleagues. Amino acids are an alternate energy source to glucose in multiple malignancies, including breast cancer. Ulaner and Schuster discuss the potential of amino acid tracers for breast cancer, including the finding that lobular breast cancers are more avid for an amino acid tracer than for FDG. Finally, imaging cell proliferation may be valuable as an early indicator of cancer therapy response. Elmi and colleagues review the development of investigational PET imaging agents for cell proliferation.

We hope this issue of PET Clinics provides an overview of the principles of breast cancer and applications of PET imaging in these patients that will improve your interpretation of PET studies and challenge the field to develop new solutions to unanswered questions.

\section{Elizabeth S. McDonald, MD, PhD, FSBI University of Pennsylvania 3400 Spruce Street Philadelphia, PA 19104-4283, USA \\ Gary A. Ulaner, MD, PhD, FACNM Department of Radiology Memorial Sloan Kettering Cancer Center 1275 York Avenue, Box 77 New York, NY, 10065, USA \\ Department of Radiology Weill Cornell Medical School New York, NY, 10065, USA \\ E-mail addresses: \\ Elizabeth.Mcdonald@uphs.upenn.edu \\ (E.S. McDonald) ulanerg@mskcc.org (G.A. Ulaner)}

\section{REFERENCE}

1. Kim CK, Zukotynski KA. Desirable properties of radiopharmaceuticals for sentinel node mapping in patients with breast cancer given the paradigm shift in patient management. Clin Nucl Med 2017;42(4): 275-9. 